\title{
ANÁLISE DA EVOLUÇÃO MICROESTRUTRAL NA LAMINAÇÃO DE TIRAS A QUENTE USANDO MÉTODO DOS ELEMENTOS FINITOS*
}

\author{
Antonio Lourenço Batista de Souza ${ }^{1}$ \\ Oscar Balancin ${ }^{2}$ \\ Rodrigo Bresciani Canto ${ }^{3}$
}

\section{Resumo}

A simulação da evolução microestrutural, durante a etapa de acabamento, na laminação de tiras a quente de aços carbono, através do software DEFORM ${ }^{\mathrm{TM}} 3 \mathrm{D}$, foi estudada para prever a carga de laminação, distribuição de temperatura, de deformação e de tamanho de grão. A boa concordância dos valores da carga e temperatura na simulação com os valores da literatura, mostra que o presente modelo é viável para representar o processo de laminação. A deformação acumulada próximo à superfície foi maior quando comparada a da região central da tira laminada, indicando uma distribuição heterogênea da deformação, que diminui com a evolução do processo. O aumento da fração de recristalização dinâmica (DRX) e a diminuição do tamanho de grão foram mais significativos nos primeiros passes. Além disso, houve uma fração de DRX maior e um tamanho de grão menor próximo a superfície da tira, esta diferença no tamanho de grão, no sentido da espessura, também diminuiu com a evolução do processo. Estes resultados sugerem que múltiplos passes podem diminuir a não homogeneidade microestrutural da tira. Assim, este estudo mostra a eficácia do modelo microestrutural em predizer o comportamento do material em um processo de laminação a quente.

Palavras-chave: Laminação de tiras a quente; Simulação numérica; DEFORM-3D; Evolução microestrutural

\section{ANALYSIS OF MICROSTRUCTURAL EVOLUTION IN HOT STRIP ROLLING USING FINITE ELEMENT METHOD}

\section{Abstract}

The microstructural evolution simulation during finish hot strip rolling of carbon steels using DEFORM ${ }^{\mathrm{TM}} 3 \mathrm{D}$ software was studied to predict the rolling load, temperature, strain and grain size distribution. The good agreement of the predicted load and temperature values of the simulation matched with the literature data shows that present modeling are feasible for the reproduce of the rolling process. The strain accumulated near the surface was larger than that in the center part, indicating the inhomogeneous distribution of strain in the rolled strip, this difference decreased with increasing rolling pass. The increase in dynamic recrystallization (DRX) fraction and the decrease in grain size were more significant in the first passes. Additionally, there was a larger DRX fraction and a smaller grain size near the strip surface, the difference of grain size, in the thickness direction, also decreased with increasing rolling pass. All these results suggest that the multi-pass hot rolling may reduce the microstructure inhomogeneity of rolled strip. Thus, this study shows the effectiveness of microstructural model in predicting the material behavior in a hot rolling process.

Keywords: Hot strip rolling; Numerical simulation; DEFORM-3D; Microstructure evolution

1 Engenheiro de Materiais, Mestrando em Ciências e Engenharia de Materiais, PPG-CEM, UFSCar, São Carlos, SP, Brasil.

2 Físico, Doutor em Engenharia Metalúrgica e de Materiais, Professor Adjunto, DEMa/PPG-CEM, UFSCar, São Carlos, SP, Brasil.

3 Engenheiro Mecânico, Doutor em Engenharia Mecânica, Professor Adjunto, DEMa, UFSCar, São Carlos, SP, Brasil.

\footnotetext{
* Contribuição técnica ao $51^{\circ}$ Seminário de Laminação - Processos e Produtos Laminados e Revestidos, 28 a 31 de outubro de 2014, Foz do Iguaçu, PR, Brasil.
} 


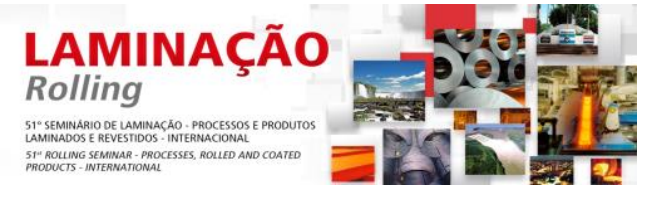

\section{INTRODUÇÃO}

O processo de conformação a quente consiste, inicialmente, em aquecer o material até a temperatura de encharque para a austenitização, em seguida, aplicar as deformações nas etapas de desbaste e de acabamento e, por fim, promover 0 resfriamento controlado do material. Além das variações dimensionais, durante o processamento ocorrem oscilações microestruturais que dependem das características do material e dos parâmetros operacionais como a deformação, a temperatura, o tempo entre passes e a taxa de deformação [1]. A evolução do grão austenítico é um dos fenômenos da metalurgia física presente no processo de laminação de tiras a quente (LTQ), cujo padrão de evolução consiste, principalmente, em quatro tipos de mecanismos, ou seja, recristalização dinâmica (DRX) durante a deformação a quente, recristalização estática (SRX) e recristalização metadinâmica (MDRX) após a deformação, bem como o crescimento de grão após a completa recristalização quando as recuperações dinâmica e estática são ignoradas. A DRX envolve a nucleação e o crescimento de novos grãos quando a densidade de discordâncias, durante a deformação, excede um valor crítico. A MDRX é a continuação do crescimento dos núcleos recristalizados dinamicamente quando a deformação é interrompida. A SRX envolve a nucleação e o crescimento de novos grãos livres de deformação, preferencialmente, em zonas de alta densidade de discordâncias. Estas modificações não só afetam, diretamente, a resistência à deformação do metal, mas também, em grande medida, determinam a microestrutura e propriedades do produto final.

Ao longo dos anos, vários autores [2-5] têm desenvolvido equações que relacionam a evolução do tamanho de grão austenítico com os parâmetros de laminação a quente. No presente trabalho serão utilizadas as equações estabelecidas por Hodgson e Gibbs [6] para o estudo da SRX e da MDRX, já no estudo da DRX serão utilizadas as equações de Yada e Senuma [7]

O método dos elementos finitos (MEF), poderosa ferramenta na simulação de processos de conformação, tem sido amplamente aplicado na modelagem do processo de LTQ por muitos autores [8-14]. No início da década de 90, Pietrzyk [15] começou a prever as distribuições de tamanho de grãos e fração recristalizada em LTQ através da combinação do MEF com modelos metalúrgicos. Posteriormente, Karhausen e Kopp [16] e Nanba et al. [17] também propuseram os seus modelos para a simulação da LTQ, no qual a evolução microestrutural foi levada em consideração. No entanto, seus trabalhos focaram na simulação de um único passe, e o processo de laminação contínua em múltiplos passes não foi considerado. Em seguida, um modelo para o processo de laminação com dois passes, considerando a cinética de recristalização, foi estabelecido e simulado por Liu e Lin [18], com base no software comercial ABAQUS. Depois disso, o problema na simulação de vários passes de laminação foi estudado por Zhou [19] e Dyja e Korczak [20], que desenvolveram alguns modelos integrados do processo de laminação e, ao mesmo tempo, obtiveram a distribuição do tamanho de grãos. No entanto, apesar da distribuição de tamanho de grão ter sido obtida nestas referências, algumas condições de laminação foram assumidas pelos autores, e a verificação dos resultados de simulação não foi completamente apresentada em algum sentido. Sendo assim, neste trabalho propõe-se uma abordagem levando-se em conta os processos de deformação e transferência de calor em um modelo capaz de fazer uma conexão entre as variáveis do processo e a microestrutura.

\footnotetext{
* Contribuição técnica ao $51^{\circ}$ Seminário de Laminação - Processos e Produtos Laminados e Revestidos, 28 a 31 de outubro de 2014, Foz do Iguaçu, PR, Brasil.
} 


\section{MATERIAIS E MÉTODOS}

Neste trabalho foi utilizada a equação constitutiva [21] (Equação 1) para o comportamento plástico do material. Para a análise da evolução microestrutural foi utilizado o modelo de Avrami e as equações expostas na Tabela 1, após desenvolvimento matemático, foram utilizadas para obterem-se os parâmetros necessários de acordo com as equações do modelo apresentadas pelo programa. As propriedades térmicas do material (condutividade térmica, capacidade calorífica e emissividade) foram copiadas da biblioteca do programa.

$$
\dot{\varepsilon}=5,5 \cdot 10^{15} \operatorname{senh}\left(0,013 \sigma_{p}\right)^{5} \exp \left(\frac{358000}{R T}\right)^{-1}
$$

Tabela 1. Equações que descrevem a evolução microestrutural dos grãos austeníticos [6,7]

\begin{tabular}{|c|c|}
\hline Deformação crítica & $\varepsilon_{c}=4,76 \cdot 10^{-4} \exp \left(\frac{8000}{T}\right)$ \\
\hline DRX & $X=1-\exp \left[-0,693\left(\frac{\varepsilon-\varepsilon_{c}}{\varepsilon_{0,5}}\right)^{2}\right]$ \\
& $\begin{array}{c}\varepsilon_{0,5}=1,144 \cdot 10^{-5} d_{0}^{0,28} \dot{\varepsilon}^{0,05} \exp \left(\frac{6420}{T}\right) \\
d_{D R X}=1,6 \cdot 10^{4} \cdot Z^{-0,23}\end{array}$ \\
\hline MDRX & $X=1-\exp \left[-0,693\left(\frac{t}{t_{0,5}}\right)^{1,5}\right]$ \\
& $\begin{array}{c}t_{0,5}=1,1 \cdot Z^{-0,8} \exp \left(\frac{240000}{R T}\right) \\
d_{M D R X}=2,6 \cdot 10^{4} \cdot Z^{-0,23}\end{array}$ \\
\hline SRX & $X=1-\exp \left[-0,693\left(\frac{t}{t_{0,5}}\right)^{1}\right]$ \\
& $\begin{array}{c}t_{0,5}=2,3 \cdot 10^{-15} \varepsilon^{-2,5} d_{0}^{2} \exp \left(\frac{230000}{R T}\right) \\
d_{S R X}=343 \varepsilon^{-0,5} d_{0}^{0,4} \exp \left(\frac{-45000}{R T}\right)\end{array}$ \\
\hline Crescimento de grão & $d^{7}=d_{0}^{7}+1,45 \cdot 10^{27} \operatorname{texp}\left(\frac{-400000}{R T}\right)$ \\
\hline
\end{tabular}

A primeira etapa do trabalho envolve a caracterização do processo industrial estudado. Nesta etapa foram utilizados os parâmetros industriais obtidos na literatura [22]. De posse destes dados, inserem-se os mesmo no software e procedese a simulação. Devido à simetria do processo de LTQ, a simulação pode ser determinada pela modelagem de um quarto da tira (metade da largura e metade da espessura) como está esquematizado na Figura 1. O número de elementos inicial na tira foi de 7200 elementos. Adicionalmente, o critério de remalhamento automático foi adotado, em vista da possibilidade de surgir malhas severamente distorcidas, causada pela extensa deformação durante a LTQ.

Os controles de simulação consistem nas informações gerais que o programa utiliza para identificar o problema, no qual são definidos os parâmetros de iteração e as condições do processo. Estes dados estão listados na Tabela 2.

* Contribuição técnica ao $51^{\circ}$ Seminário de Laminação - Processos e Produtos Laminados e Revestidos, 28 a 31 de outubro de 2014, Foz do Iguaçu, PR, Brasil. 


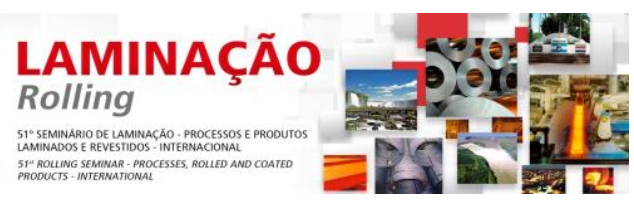

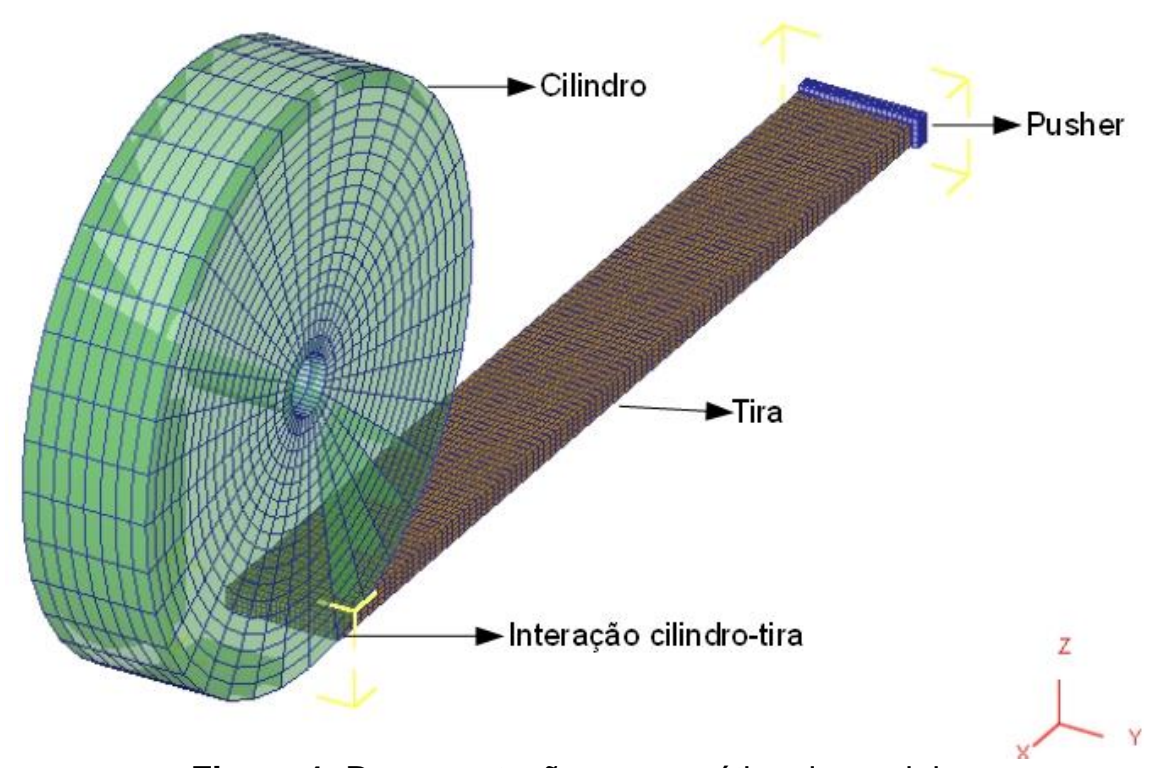

Figura 1. Representação esquemática do modelo.

Tabela 2. Dados de entrada para o controle de simulação

\begin{tabular}{|l|l|}
\hline Principal \\
\hline $\begin{array}{l}\text { Sistema de Unidades } \\
\text { Tipo de Simulação } \\
\text { Modos de Simulação }\end{array}$ & $\begin{array}{l}\text { SI } \\
\text { Lagrangeano Incremental } \\
\text { Deformação, Transferência de Calor (Grão) }\end{array}$ \\
\hline Iteração \\
\hline Solucionador Deformação & Esparso \\
Método de Iteração & Iteração Direta \\
Solucionador Temperatura & Esparso \\
Erro Limite Velocidade & 0,01 \\
Erro Limite Força & 0,05 \\
\hline Condições de Processo \\
\hline Temperatura Ambiente & $30^{\circ} \mathrm{C}$ \\
Coeficiente de Convecção & $0,02 \mathrm{~N}^{-1} \mathrm{~s}^{-1} \cdot \mathrm{mm}^{-1} \cdot{ }^{\circ} \mathrm{C}^{-1}$ \\
\hline
\end{tabular}

No caso da laminação, os objetos são a tira e os cilindros do laminador. Neste trabalho os cilindros foram definidos com o comportamento rígido e a tira com comportamento plástico. Continuando na definição dos objetos, são estabelecidos os parâmetros de temperatura inicial dos objetos. As temperaturas iniciais dos cilindros foram definidas em $40^{\circ} \mathrm{C}$ e a da tira em $987^{\circ} \mathrm{C}$. Os planos de simetria são definidos de acordo com a geometria dos objetos e as áreas de transferência de calor correspondem às faces que estão expostas ao ambiente.

A interação entre objetos é importante para definir como os objetos interagem entre si. As interações são mecânicas, como o atrito, e também são térmicas, como a transferência de calor por condução. Os parâmetros de interação utilizados estão na Tabela 3.

Tabela 3. Dados de entrada para interação entre objetos

\begin{tabular}{|l|c|}
\hline & Interação Cilindro-Tira \\
\hline Tipo de Atrito & Cisalhante \\
Coeficiente de Atrito & 0,7 \\
Coef. Transf. de Calor $\left(\mathrm{N}^{-1} \mathrm{~s}^{-1} \cdot \mathrm{mm}^{-1} \cdot{ }^{\circ} \mathrm{C}^{-1}\right)$ & 40 \\
\hline
\end{tabular}

* Contribuição técnica ao $51^{\circ}$ Seminário de Laminação - Processos e Produtos Laminados e Revestidos, 28 a 31 de outubro de 2014, Foz do Iguaçu, PR, Brasil. 


\section{RESULTADOS E DISCUSSÃO}

\subsection{Carga e Temperatura de Laminação}

Na Figura 2a é apresentada a comparação entre o valor da carga de laminação calculado e o obtido na literatura, durante o processo industrial. A boa aproximação dos valores simulados com os valores obtidos da literatura, mostra que o presente modelo é viável para representar o processo de laminação. A evolução da temperatura na superfície da tira, durante a simulação da LTQ, foi comparada com a temperatura dos dados obtidos na literatura, como mostrado na Figura 2b, mostrando uma pequena variação. Esta variação pode ser atribuída, provavelmente, à formação de uma fina camada de óxido na superfície quente da tira, uma vez que esta não é considerada na simulação.

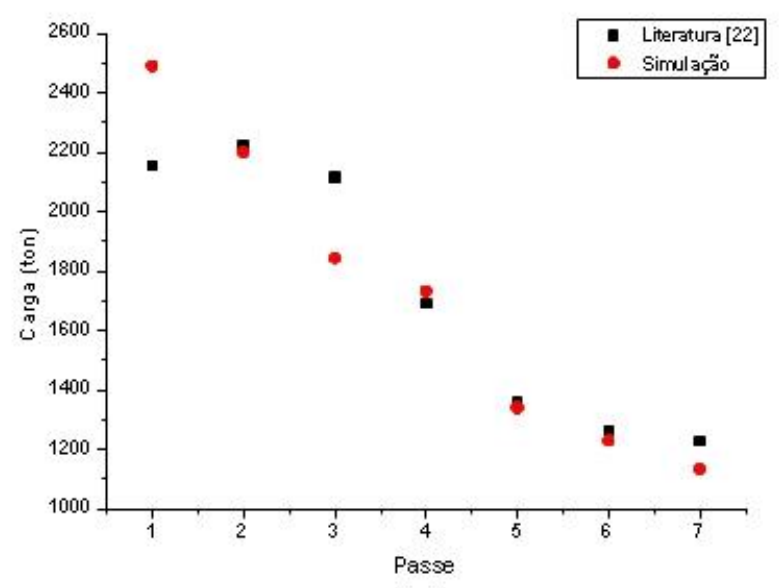

(a)

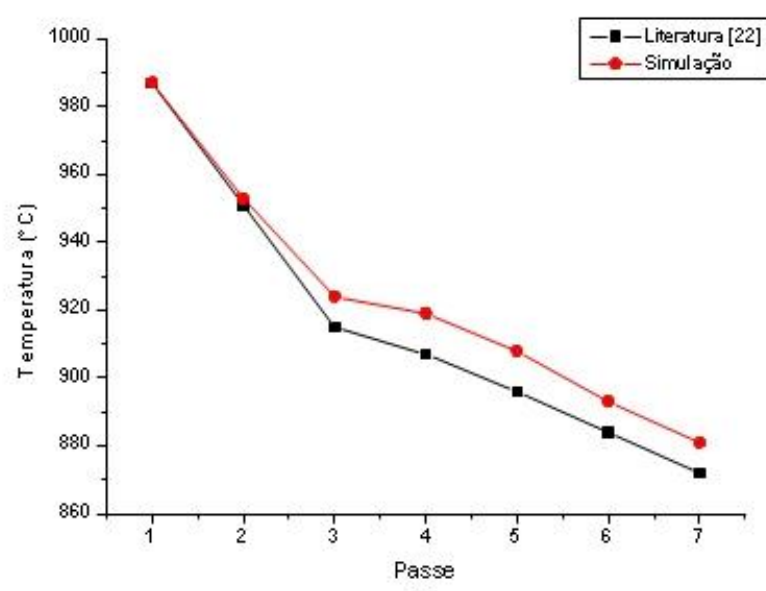

(b)

Figura 2. Gráfico (a) da carga e (b) da temperatura de laminação, com os valores da simulação e os obtidos na literatura [22], para os diversos passes do processo.

\subsection{Perfil de Deformação}

As variações de deformação na tira podem afetar diretamente a ocorrência dos mecanismos de recristalização e a variação das frações recristalizadas e do tamanho de grão. Sendo assim, a análise da deformação aplicada contribui para o completo entendimento da evolução microestrutural durante o processo. De acordo com o que é apresentado na Figura 3, em que é mostrado um quarto da seção transversal da tira em cada passe, a deformação imposta próximo à superfície da tira foi maior do que a imposta na região central, o que indica uma distribuição não uniforme da deformação na tira laminada, podendo gerar uma microestrutura não homogênea. No entanto, observa-se uma deformação menos uniforme nos primeiros passes de laminação, quando comparados com os últimos passes, nos quais as deformações aplicadas são menores. Sendo assim, uma possível maneira de reduzir esta não uniformidade seria realizar o processo com maior número de passes.

\footnotetext{
* Contribuição técnica ao $51^{\circ}$ Seminário de Laminação - Processos e Produtos Laminados e
} Revestidos, 28 a 31 de outubro de 2014, Foz do lguaçu, PR, Brasil. 

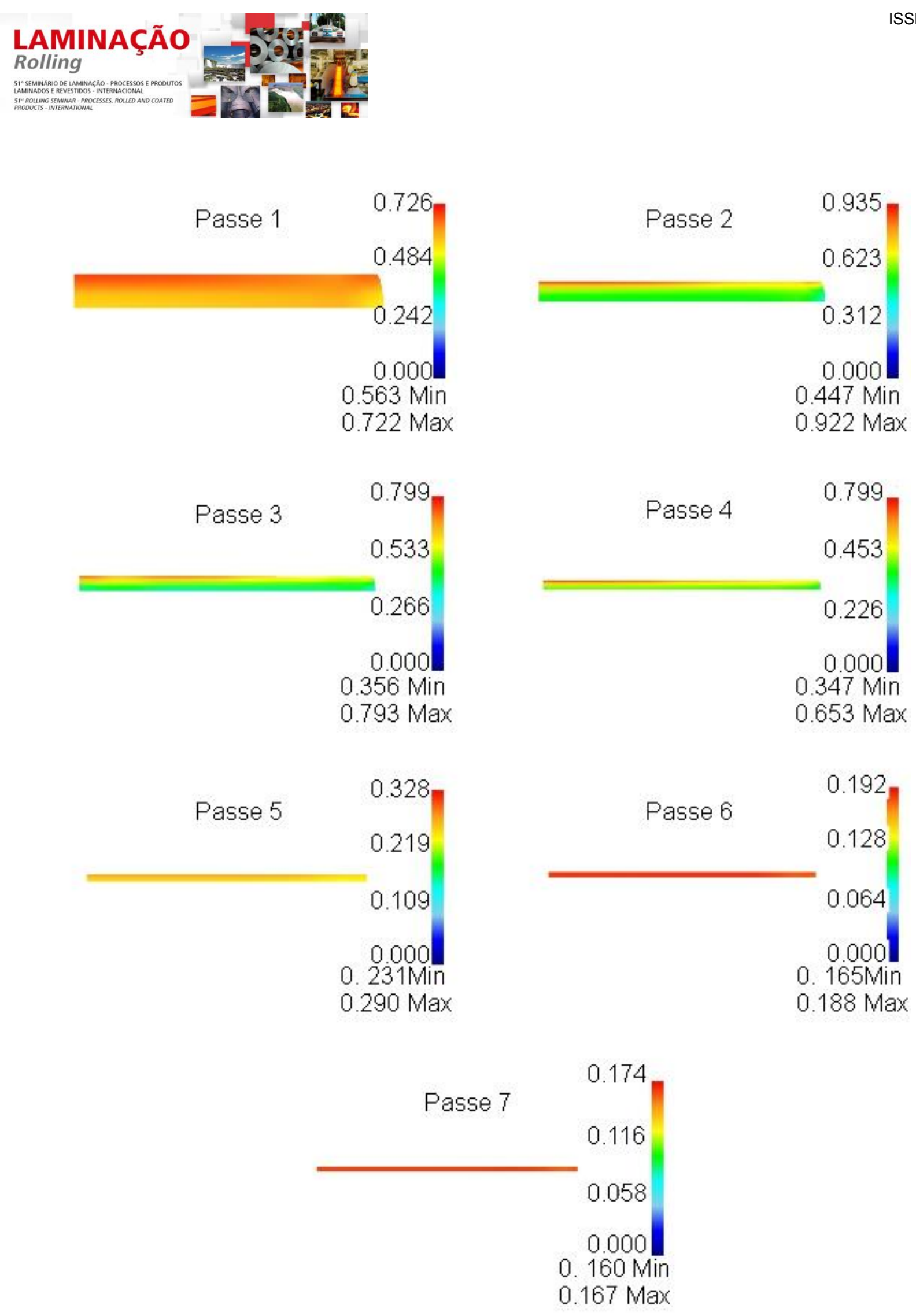

Figura 3. Perfil de deformação efetiva, ao longo da espessura da tira, em cada passe.

\subsection{Recristalização Dinâmica}

Devido à alta velocidade do processo, a DRX não é completa. Portanto, os núcleos gerados durante a aplicação de deformação vão crescer continuamente através da MDRX após deformação. Enquanto isso, os núcleos de SRX vão nuclear e começar a desenvolver-se simultaneamente no intervalo entre passes (Figura 4). Como a

\footnotetext{
* Contribuição técnica ao $51^{\circ}$ Seminário de Laminação - Processos e Produtos Laminados e Revestidos, 28 a 31 de outubro de 2014, Foz do Iguaçu, PR, Brasil.
} 
taxa de DRX é maior do que a de SRX e MDRX, ocorre um acentuado refino de grão nos três primeiros passes. Sendo assim, nota-se que a DRX influencia a cinética de transformação e microestrutura recristalizada de forma mais acentuada do que a SRX durante a deformação a quente.

DRX

1.00

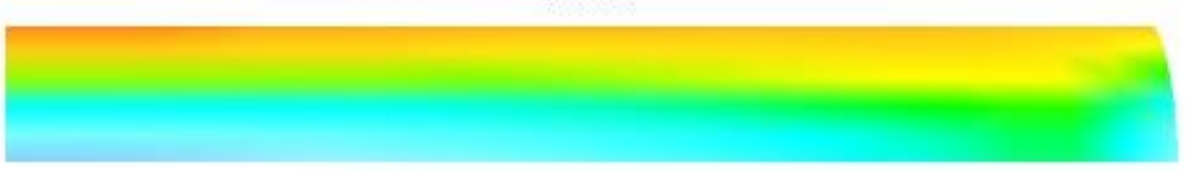

0.667

MDRX

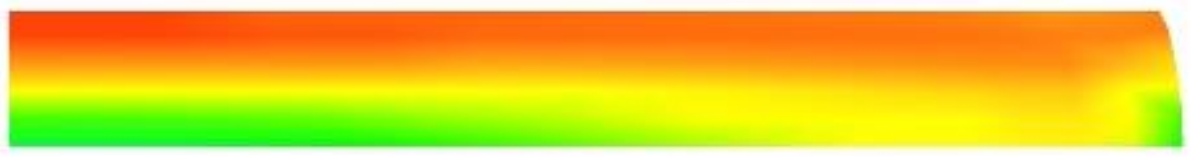

SRX

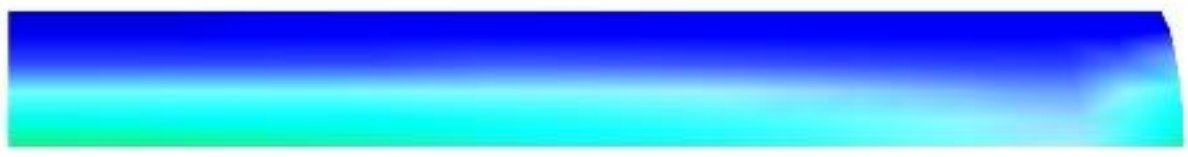

0.000

Figura 4. Perfil de fração recristalizada, ao longo da espessura da tira, para o primeiro passe e intervalo entre passe.

O perfil de fração de DRX em cada passe do processo de laminação, em que é mostrado um quarto da seção transversal da tira em cada passe, pode ser visto na Figura 5. Observa-se que a DRX ocorre de maneira mais expressiva nos três primeiros passes, tornando-se quase completa no terceiro passe. Nos quatro últimos passes, a DRX ocorre parcialmente, por causa da queda da temperatura de laminação e menores deformações aplicadas.

Na Figura 5 observa-se, também, uma distribuição heterogênea da fração de DRX ao longo da espessura da tira, tendo uma fração de DRX maior próximo à superfície, que está relacionado com a presença de maiores deformações nestas regiões. Assim como ocorre com o perfil de deformação, a fração de DRX se torna mais uniforme ao longo dos passes do processo.

* Contribuição técnica ao $51^{\circ}$ Seminário de Laminação - Processos e Produtos Laminados e Revestidos, 28 a 31 de outubro de 2014, Foz do Iguaçu, PR, Brasil. 
Passe 1

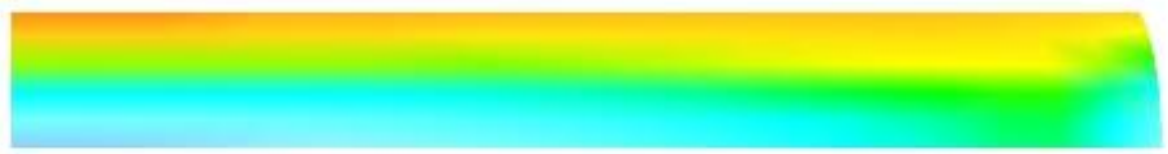

Fraçäo DRX

1.00

Passe 2

Passe 3

Passe 5

Passe 6

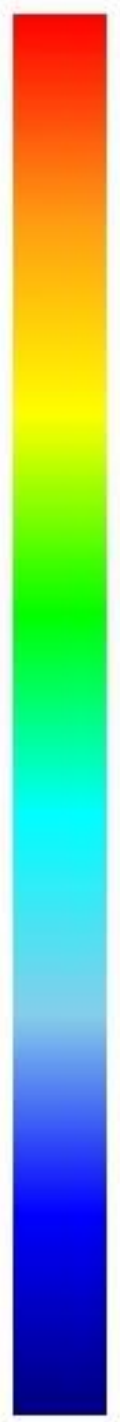

\section{Passe 7}

Figura 5. Perfil de fração de DRX, ao longo da espessura da tira, em cada passe.

\subsection{Tamanho de Grão Austenítico}

A evolução da microestrutura é calculada em cada elemento da malha traçada para a tira. Um tamanho de grão austenítico inicial de $100 \mu \mathrm{m}$ foi assumido para todos os elementos. A evolução do tamanho de grão também foi comparada para dois pontos diferentes, um na superfície e outro no centro da tira, como mostrado na Figura 6 . 0 tamanho de grão cai rapidamente na fase inicial, nos primeiros dois passes. Esta

* Contribuição técnica ao $51^{\circ}$ Seminário de Laminação - Processos e Produtos Laminados e Revestidos, 28 a 31 de outubro de 2014, Foz do Iguaçu, PR, Brasil. 


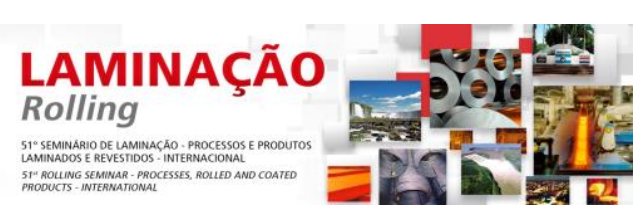

múltiplos passes de laminação a quente é influenciada, principalmente, pela deformação (e taxa de deformação) e temperatura.

Apesar de a microestrutura recristalizada durante os três primeiros passes apresentar um refinamento de grão significativo, a distribuição do tamanho de grão é mais heterogênea, no sentido da espessura da tira, em relação aos quatro últimos passes. Estes resultados sugerem que múltiplos passes podem auxiliar na maior homogeneidade microestrutural da tira.

Outro resultado de interesse é que a deformação aplicada nos primeiros passes é suficiente para que ocorra a $\mathrm{DRX}$, principalmente na região próxima à superfície da tira, tornando-se quase completa em toda a tira no terceiro passe. Subsequentemente, há uma redução da DRX nos últimos passes, sendo a fração recristalizada no último passe em torno de $55 \%$.

O tamanho de grão diminui do valor inicial de $100 \mu \mathrm{m}$ para o valor final de $11,5 \mu \mathrm{m}$ após os sete passes de laminação. A predição é razoável em relação aos produtos do mercado de tiras laminadas.

Espera-se que tal modelagem possa ser utilizada como um pacote para o software comercial para prever as características microestruturais e fornecer uma visão sobre a evolução da microestrutura durante a laminação industrial a quente.

\section{Agradecimentos}

Os autores agradecem ao suporte financeiro dado pelo Conselho Nacional de Desenvolvimento Científico e Tecnológico - CNPq (Processo: 131427/2013-0).

\section{REFERÊNCIAS}

1 Regone W. Simulação da laminação a quente de um aço livre de intersticiais IF através de ensaios de torção [Tese de Doutorado]. São Carlos, Brasil: Universidade Federal de São Carlos; 2001.

2 Sellars C, Whiteman J. Recrystallization and grain growth in hot rolling. Metal Science. 1979; 13(3-4): 3-4.

3 Siciliano Jr F, Minami K, Maccagno T, Jonas JJ. Mathematical modeling of the mean flow stress, fractional softening and grain size during the hot strip rolling of C-Mn steels. ISIJ international. 1996; 36(12): 1500-1506.

4 Maccagno T, Jonas JJ, Hodgson P. Spreadsheet modelling of grain size evolution during rod rolling. ISIJ international. 1996; 36(6): 720-728.

5 Siciliano Jr F, Jonas JJ. Mathematical modeling of the hot strip rolling of microalloyed $\mathrm{Nb}$, multiply-alloyed $\mathrm{Cr}-\mathrm{Mo}$, and plain C-Mn steels. Metallurgical and materials transactions A. 2000; 31(2): 511-530.

6 Hodgson P, Gibbs R. A mathematical model to predict the mechanical properties of hot rolled C-Mn and microalloyed steels. ISIJ international. 1992; 32(12): 1329-1338.

7 Senuma T, Yada H. Microstructural evolution of plain carbon steels in multiple hot working. In Proceedings of the Riso International Symposium on Metallurgy and Materials Science, 7th; 1986; RISO National Laboratory, Roskilde, Dinamarca. p. 547552.

8 Wang MT, Zang XL, Li XT, Du FS. Finite element simulation of hot strip continuous rolling process coupling microstructural evolution. Journal of Iron and Steel Research, International. 2007; 14(3): 30-36.

9 Kim J, Lee J, Hwang SM. An analytical model for the prediction of strip temperatures in hot strip rolling. International Journal of Heat and Mass Transfer. 2009; 52(7): 18641874.

\footnotetext{
* Contribuição técnica ao $51^{\circ}$ Seminário de Laminação - Processos e Produtos Laminados e Revestidos, 28 a 31 de outubro de 2014, Foz do Iguaçu, PR, Brasil.
} 
10 Ding H, Hirai K, Homma T, Kamado S. Numerical simulation for microstructure evolution in AM50 Mg alloy during hot rolling. Computational Materials Science. 2010; 47(4): 919-925.

11 Ding HI, Wang Ty, Yang L, Kamado S. FEM modeling of dynamical recrystallization during multi-pass hot rolling of AM50 alloy and experimental verification. Transactions of Nonferrous Metals Society of China. 2013; 23(9): 2678-2685.

12 Wang X, Li F, Yang Q, He A. FEM analysis for residual stress prediction in hot rolled steel strip during the run-out table cooling. Applied Mathematical Modelling. 2013; 37(1): 586-609.

13 Nalawade R, Puranik A, Balachandran G, Mahadik K, Balasubramanian V. Simulation of hot rolling deformation at intermediate passes and its industrial validity. International Journal of Mechanical Sciences. 2013; 77: 8-16.

14 Qingqiang H, Jia S, Chengxin Y, Junyou Z, Zongbo Z. Thermo-mechanical modeling and simulation of microstructure evolution in multi-pass $\mathrm{H}$-shape rolling. Finite Elements in Analysis and Design. 2013; 76: 13-20.

15 Pietrzyk M. Finite element based model of structure development in the hot rolling process. Steel research. 1990; 61(12): 603-607.

16 Karhausen K, Kopp R. Model for integrated process and microstructure simulation in hot forming. Steel Research. 1992; 63(6): 247-256.

17 Nanba S, Kitamura M, Shimada M, Katsumata M, Inoue T, Imamura H, et al. Prediction of microstructure distribution in the through-thickness direction during and after hot rolling in carbon steels. ISIJ international. 1992; 32(3): 377-386.

18 Liu Y, Lin J. Modelling of microstructural evolution in multipass hot rolling. Journal of materials processing technology. 2003; 143: 723-728.

19 Zhou S. An integrated model for hot rolling of steel strips. Journal of Materials Processing Technology. 2003; 134(3): 338-351.

20 Dyja H, Korczak P. The thermal-mechanical and microstructural model for the FEM simulation of hot plate rolling. Journal of Materials Processing Technology. 1999; 92: 463-467.

21 Cutrim, R.M. Influência das Condições de Deformação na Recristalização Dinâmica do Aço Médio Carbono 38MnSiVS5 Microligado ao Vanádio [Dissertação de Mestrado]. São Carlos, Brasil: Universidade Federal de São Carlos, 2009.

22 Siciliano Jr F. Mathematical Modeling of the Hot Strip Rolling of Nb Microalloyed Steels [PhD Thesis]. Montreal, Canada: McGill University; 1999.

* Contribuição técnica ao $51^{\circ}$ Seminário de Laminação - Processos e Produtos Laminados Revestidos, 28 a 31 de outubro de 2014, Foz do Iguaçu, PR, Brasil. 